\title{
Heparin-binding protein targeted to mitochondrial compartments protects endothelial cells from apoptosis
}

\author{
A. Maria Olofsson, ${ }^{1}$ Mikael Vestberg, ${ }^{1}$ Heiko Herwald, ${ }^{1}$ Jørgen Rygaard, ${ }^{2}$ Guido David, ${ }^{3}$ \\ Karl-E. Arfors, ${ }^{4}$ Viggo Linde, ${ }^{5}$ Hans Flodgaard, ${ }^{5}$ Jürgen Dedio, ${ }^{6}$ Werner Müller-Esterl, ${ }^{6}$ \\ and Evy Lundgren-Åkerlund ${ }^{1}$
}

\author{
${ }^{1}$ Department of Cell and Molecular Biology, Lund University, S-22100 Lund, Sweden \\ ${ }^{2}$ Bartholin Institute, Kommunehospitalet, DK-1399 Copenhagen, Denmark \\ ${ }^{3}$ Center for Human Genetics, Flanders Interuniversity Institute for Biotechnology, University of Leuven, \\ B-3000 Leuven, Belgium \\ ${ }^{4}$ Experimental Medicine Inc., Princeton, New Jersey 08540, USA \\ ${ }^{5}$ Novo Nordisk A/S, DK-2880 Bagsvaerd, Denmark \\ ${ }^{6}$ Institute for Physiological Chemistry and Pathobiochemistry, Johannes Gutenberg University at Mainz, \\ D-55099 Mainz, Germany
}

Address correspondence to: Werner Müller-Esterl, Institute for Physiological Chemistry and Pathobiochemistry, Johannes Gutenberg University at Mainz, Duesbergweg 6, D-55099 Mainz, Germany.

Phone: 49-6131-395890; Fax: 49-6131-395792; E-mail: wme@mail.uni-mainz.de.

Received for publication March 1, 1999, and accepted in revised form August 13, 1999.

\begin{abstract}
Neutrophil-borne heparin-binding protein (HBP) is a multifunctional protein involved in the progression of inflammation. HBP is stored in neutrophil granules and released upon stimulation of the cells in proximity to endothelial cells. HBP affects endothelial cells in multiple ways; however, the molecular and cellular mechanisms underlying the interaction of HBP with these cells are unknown. Affinity isolation and enzymatic degradation demonstrated that HBP released from human neutrophils binds to endothelial cell-surface proteoglycans, such as syndecans and glypican. Flow cytometry indicated that a significant fraction of proteoglycan-bound HBP is taken up by the endothelial cells, and we used radiolabeled HBP to determine the internalization rate of surface-bound HBP. Confocal and electron microscopy revealed that internalized HBP is targeted to perinuclear compartments of endothelial cells, where it colocalizes with mitochondria. Western blotting of isolated mitochondria from HBP-treated endothelial cells showed that HBP is present in 2 forms -28 and $22 \mathrm{kDa}$. Internalized HBP markedly reduced growth factor deprivation-induced caspase-3 activation and protected endothelial cells from apoptosis, suggesting that uptake and intracellular routing of exogenous HBP to mitochondria contributes to the sustained viability of endothelial cells in the context of locally activated neutrophils.
\end{abstract}

J. Clin. Invest. 104:885-894 (1999).

\section{Introduction}

The interplay between circulating white blood cells and the vascular endothelium has attracted considerable interest over the past decade (for a recent review, see ref. 1). Polymorphonuclear neutrophils (PMNs) represent the first line of host defense against infection (2). They roll on the endothelium, probing for signs of infection. Upon sensitization by inflammatory signals, neutrophils adhere to the endothelium of postcapillary venules adjacent to the infected tissue (3). Adhesion proteins, such as selectins, integrins, members of the Ig superfamily, and proteoglycans, play pivotal roles in these processes (3). After physical attachment to the endothelium, the neutrophils extravasate, migrate to the infected area, and begin to combat the infection. In the inflamed tissue, neutrophils produce oxygen radicals and mobilize a vast array of effector proteins stored in their internal compartments, i.e., the azurophilic, specific, and gelatinase granules, as well as the secretory vesicles (4).
Among the components present in these granules, special attention has been paid to heparin-binding protein (HBP), also known as CAP37 or azurocidin (5). Originally, human HBP was studied because of its intrinsic antibiotic activities and LPS-binding properties $(6,7)$. For example, in animal models of fecal peritonitis, HBP treatment rescues mice from otherwise lethal injury (8). More recent evidence suggests that HBP, in addition to its bactericidal role, contributes to the maintenance and progression of inflammation by recruitment and activation of monocytes $(9,10)$ and mobilization of T cells (11), and by the detachment and homotypic aggregation of endothelial cells and fibroblasts in vitro (12). Hence, neutrophil-borne HBP appears to be a multifunctional protein endowed with LPS- and heparin-binding capacity (13). Despite the far-reaching implications for host defense and inflammation, the molecular mechanisms and cellular signaling pathways underlying the biological roles of HBP have remained largely unknown. 
Structurally, HBP belongs to the serprocidin subgroup of the chymotrypsin-like protease superfamily comprising neutrophil elastase, cathepsin G, and proteinase-3 (14). Unlike these latter enzymes, HBP lacks proteolytic activity owing to mutations that change 2 of the essential amino acid residues of the catalytic triad, H41S and S175G (13-15), thereby crippling the critical charge-transfer system required for peptide-bond hydrolysis. Hence, HBP represents another member of the small group of pseudoproteases that includes hepatocyte growth factor and haptoglobin. The crystal structure of human HBP has been resolved (13) and compared with that of human elastase (the most similar protein), with a $44 \%$ sequence identity $(15,16)$. Human HBP contains a serine protease fold exposing 2 charged patches, a basic area and an acidic area on each side of the protein surface, which are not present in elastase (13). The basic patch likely represents the docking site for heparin, whereas the function of the acidic patch if any - is unknown. Adjacent to its heparin binding site, HBP exposes a high-affinity binding site for lipid A, which likely mediates LPS attachment (13).

To date, no attempts have been made to characterize the fate of HBP after its secretion by neutrophils. Here we demonstrate that neutrophil-borne HBP binds to surface-exposed proteoglycans of human umbilical vein endothelial cells (HUVECs). We show that HUVECs bind, sequester, and route neutrophil-borne HBP from the cell surface to perinuclear compartments, where it colocalizes with mitochondria. Internalized HBP confers beneficial effects to endothelial cells, such as protection from growth factor deprivation-induced apoptosis.

\section{Methods}

Antibodies and proteins. Recombinant human HBP was produced using the baculovirus expression system in Sf9 insect cells (Invitrogen Corp., Carlsbad, California, USA) and was purified as described (10). Biotinylation and coupling of HBP to FITC were done as described $(17,18)$. Iodination of HBP was done with $N$-succinimidyl 3-(4-hydroxy-[ $\left.{ }^{125} \mathrm{I}\right]$ iodophenyl)propionate (Amersham International, Amersham, United Kingdom) (19). Mouse $\mathrm{mAb} 2 \mathrm{~F} 23 \mathrm{C} 3$ and rabbit antisera to recombinant HBP (anti-HBP) (20), rabbit antibody (AS385) to human mitochondrial protein $\mathrm{p} 33 / \mathrm{gC} 1 \mathrm{qR}$ (anti-p33) (21), and mouse mAb 3G10 to heparitinase-digested heparan sulfate proteoglycan, reactive with the $\Delta 4,5$ glucuronates at the nonreducing ends of the heparan sulfate chain remnants (22), were prepared and affinitypurified as detailed previously (20-22). Mouse mAb 1273 directed against $65-\mathrm{kDa}$ protein of human mitochondria (23) was from Chemicon International (Temecula, California, USA). Texas red-conjugated goat anti-rabbit IgG was from Jackson ImmunoResearch Laboratories Inc. (West Grove, Pennsylvania, USA); FITC-conjugated goat anti-mouse IgG was from Sigma Chemical Co. (St. Louis, Missouri, USA); peroxidaseconjugated antibody to rabbit IgG was from Bio-Rad
Laboratories Inc. (Richmond, California, USA); and alkaline phosphatase-conjugated rabbit anti-mouse Ig was from Promega Corp. (Madison, Wisconsin, USA). Gold-labeled goat anti-rabbit and goat anti-mouse Ig were from Amersham International.

Isolation and activation of neutrophils. Neutrophils were isolated from heparinized human blood using Macrodex sedimentation of erythrocytes and Percoll (Pharmacia Biotech AB, Uppsala, Sweden) separation of leukocyte-rich plasma (24). PMNs $\left(2 \times 10^{5}\right.$ cells $/ 200$ $\mu \mathrm{L})$ were stimulated with varying concentrations of PMA, FMLP, or leukotriene $\mathrm{B}_{4}$ for 30 minutes at $37^{\circ} \mathrm{C}$ in the presence or absence of HUVECs grown to confluence in 48-well plates. The PMN suspensions were removed and centrifuged for 10 seconds at 20,800 g, and the HBP concentration of the supernatants was determined by ELISA.

Cell culture. HUVECs were isolated from umbilical cords by digestion with collagenase (Worthington Biochemical Corp., Freehold, New Jersey, USA) and were cultured on gelatinized surfaces (Sigma Chemical Co.) in the presence of FCS and adult calf serum (1:1 vol/vol; GIBCO BRL, Paisley, Scotland), endothelial cell growth factor, heparin (Sigma Chemical Co.), and antibiotics in M199 with Earle's salt (GIBCO BRL) (25, 26). Primary cultures were passaged once (unless stated otherwise) with trypsin/EDTA. Cells were used when expressing cobblestone morphology, except for microscopic studies, where subconfluent cells were applied. For metabolic labeling, HUVECs were cultured in the presence of $100 \mu \mathrm{Ci} / \mathrm{mL}\left[{ }^{35} \mathrm{~S} \mathrm{Na}_{2} \mathrm{SO}_{4}\right.$ (specific activity: $1,310 \mathrm{Ci} / \mathrm{mmoL}$ ) in culture media. After 24 hours, the cells were washed 4 times with cold PBS and lysed on a shaker for 1 hour at $4{ }^{\circ} \mathrm{C}$ with $1 \%$ Triton $\mathrm{X}$ -

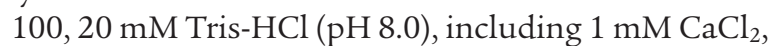
$1 \mathrm{mM} \mathrm{MgCl}_{2}, 2 \mathrm{mM}$ PMSF, $5 \mathrm{mM}$ 1,10-phenanthroline, $4 \mu \mathrm{g} / \mathrm{mL}$ leupeptin, $4 \mu \mathrm{g} / \mathrm{mL}$ pepstatin $\mathrm{A}$, and 100 $\mu \mathrm{g} / \mathrm{mL}$ aprotinin. The lysate was centrifuged at 10,000 $g$ for 30 minutes at $4^{\circ} \mathrm{C}$, and the supernatant was further processed. $\mathrm{CHO}-\mathrm{K} 1$ (from Chinese hamster ovary) and the heparan sulfate-deficient cell line CHO-K1 variant pgsD-677 (27) were grown in F12K nutrient mixture with Kaighn's modifications (GIBCO BRL) and supplemented with FCS and antibiotics.

Cell fractionation. Confluent HUVECs, grown on 6 dishes (145 $\mathrm{cm}^{2}$ each), were washed once with M199 in HBSS (GIBCO BRL). Plates were incubated with 25 $\mu \mathrm{g} / \mathrm{mL}$ of HBP in $5 \mathrm{~mL}$ of the same solution for 24 hours at $37^{\circ} \mathrm{C}$. Cells were washed once with $\mathrm{Ca}^{2+} / \mathrm{Mg}^{2+}$-free PBS (GIBCO BRL) and scraped from the plates. After centrifugation (800 $g$ for 10 minutes), cells were resuspended in $2.5 \mathrm{~mL}$ of $50 \mathrm{mM}$ phosphate $(\mathrm{pH}$ 7.4), $0.28 \mathrm{M}$ sucrose, $100 \mu \mathrm{g} / \mathrm{mL}$ PMFS, $1 \mu \mathrm{g} / \mathrm{mL}$ aprotinin, $0.5 \mu \mathrm{g} / \mathrm{mL}$ leupeptin, $1 \mu \mathrm{g} / \mathrm{mL}$ pepstatin $\mathrm{A}$, and $3.6 \mu \mathrm{g} / \mathrm{mL}$ trans-epoxylsuccinyl-L-leucylamido-(4-guanidino)butane (E-64) and pressurized with $\mathrm{N}_{2}$ for 5 minutes at $350 \mathrm{psi}$ and $4^{\circ} \mathrm{C}$. The resultant suspension was used for cell fractionation as detailed previously (21). Pellets P3 (vesicular fraction), P4 (membrane fraction), 

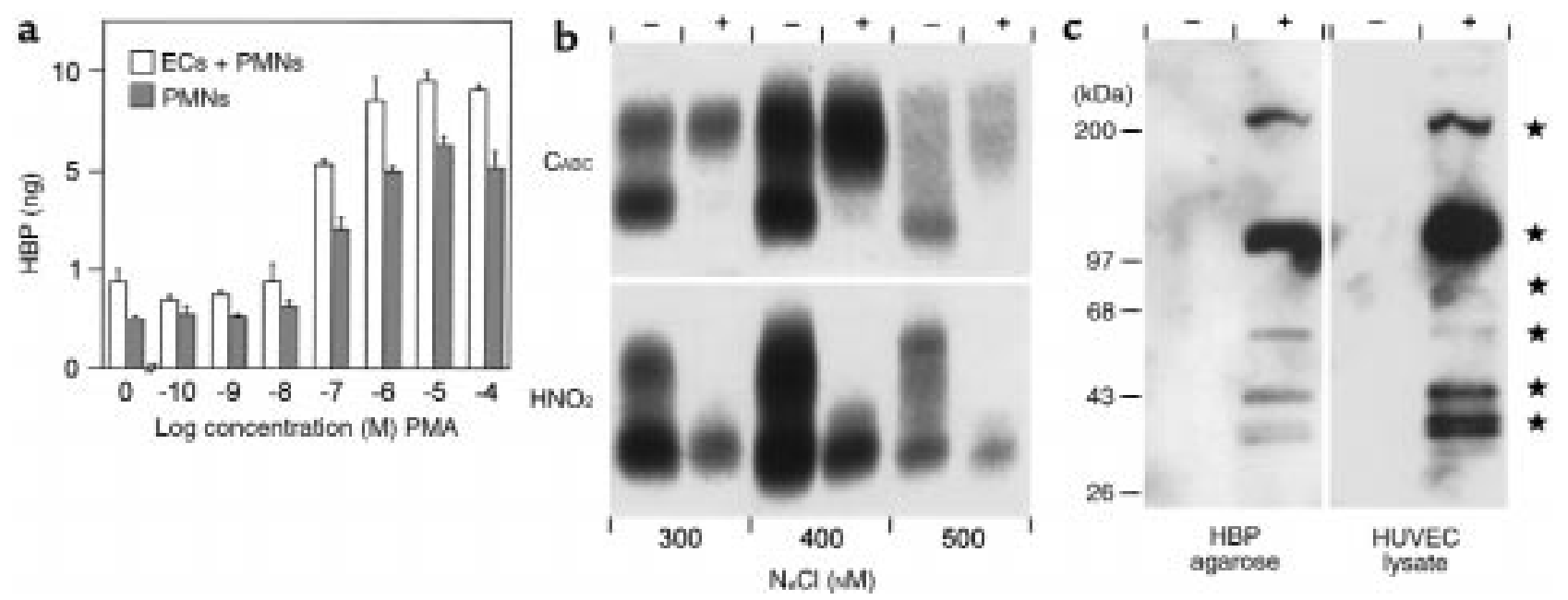

Figure 1

Release and uptake of HBP. (a) Release of HBP from activated human neutrophils was followed after stimulating isolated PMNs with the indicated concentrations of PMA in the absence (gray bars) or presence (open bars) of HUVECs (ECs) cultured in 48-well plates. After removal of the cell suspension from the plate and centrifugation, the concentration of HBP in the cell-free supernatant was determined by ELISA. Experiments were done in triplicate; means \pm SD are presented. (b) ${ }^{35}$ S-labeled HBP binding sites were isolated from whole lysates of HUVECs by affinity chromatography on HBP-streptavidin agarose. Fractions eluted at 300,400 , or $500 \mathrm{mM} \mathrm{NaCl}$ were incubated in the presence (+) or absence (-) of $\mathrm{C}_{\mathrm{ABC}}$ (top) or $\mathrm{HNO}_{2}$ (bottom). Cleavage products were separated on agarose gels and viewed by phosphoimaging. (c) Affinity-purified HBP binding sites eluted at $300 \mathrm{mM} \mathrm{NaCl}$ (left) or whole HUVEC lysates (right) were incubated in the absence (-) or presence (+) of $\mathrm{C}_{\mathrm{ABC}}$ and heparitinase. The cleavage products were separated by SDS-PAGE, electrotransferred to Zeta-Probe membranes, and probed by mAb 3G10. The relative positions of known endothelial proteoglycans of the heparan sulfate type are indicated by stars (from top to bottom): perlecan (>200 kDa), syndecan-3 (125 kDa), syndecan-1 (90 kDa), glypican (64 kDa), syndecan-2 (48 kDa), and syndecan-4 (35 kDa). The relative molecular masses of marker proteins are given on the left.

and P5 (microsomal fraction), as well as supernatant S3 (cytosolic fraction), were collected. Mitochondria were isolated from HUVECs incubated in the absence or presence of $50 \mathrm{mg} / \mathrm{mL}$ HBP for 12 hours by differential ultracentrifugation (28). Aliquots of the eluted fractions were subjected to $4-16 \%$ PAGE in the presence of SDS (29) and 5\% 2-mercaptoethanol. Immunoblotting was done with polyclonal anti-HBP (from rabbit) and the chemiluminescence detection method.

Isolation and identification of proteoglycans. HBP binding sites were isolated from the lysates of ${ }^{35}$ S-labeled HUVECs by affinity chromatography on biotinylated HBP-streptavidin agarose. Proteoglycans were isolated from the eluted material by the Alcian blue method (30). A fraction of the sample was treated with $\mathrm{HNO}_{2}$ at $\mathrm{pH} 1.5$ for 10 minutes at room temperature to cleave heparan sulfate (31). Another fraction was dissolved in $50 \mathrm{mM}$ Tris- $\mathrm{HCl}, 0.1 \mathrm{M} \mathrm{NaCl}$ at $\mathrm{pH} 7.3$, and digested with $150 \mathrm{mU} / \mathrm{mL}$ chondroitinase ${ }_{A B C}\left(C_{A B C}\right.$; Sigma Chemical Co.) (30). Samples were run on $1.2 \%$ agarose gels (32). For Western blotting, affinity-purified material from HBP-streptavidin agarose was concentrated on $100 \mu \mathrm{L}$ DEAE-Trisacryl columns (BioSepra SA, Villeneuve-la-Garenne, France) and eluted with buffer A containing $1 \mathrm{M} \mathrm{NaCl}(5 \times 50 \mu \mathrm{L})$. Samples were digested for 3 hours at $37^{\circ} \mathrm{C}$ with $0.5 \mathrm{U} / \mathrm{mL} \mathrm{C}_{\mathrm{ABC}}$ (Seikagaku Corp., Tokyo, Japan) or $10 \mathrm{mU} / \mathrm{mL}$ heparitinase (Seikagaku Corp.) in $100 \mathrm{mM} \mathrm{NaCl}, 1 \mathrm{mM} \mathrm{CaCl} 2,0.1 \%$ Triton $\mathrm{X}-100,50 \mathrm{mM}$ 6-aminohexanoic acid, $20 \mu \mathrm{g} / \mathrm{mL}$ leupeptin, $2.5 \mu \mathrm{g} / \mathrm{mL}$ pepstatin A, $1 \mathrm{mM}$ PMSF, and 50
mM HEPES at $\mathrm{pH}$ 7.0. After 6-20\% SDS-PAGE under nonreducing conditions, separated proteins were transferred to Zeta-Probe membranes (Bio-Rad Laboratories Inc.). Immunoblotting was done with antibody $3 \mathrm{G} 10$, followed by rabbit anti-mouse Ig conjugated to alkaline phosphatase $(1: 5,000)$. Bound antibody was viewed using the chemiluminescence method.

Flow cytometry. HUVECs grown to confluence in 12-well plates were washed once with M199/HBSS and incubated for various periods of time at $37^{\circ} \mathrm{C}$ with $50 \mu \mathrm{g} / \mathrm{mL}$ of HBP in the same medium; controls were processed in the absence of HBP. The cells were washed twice with PBS containing $0.5 \%$ HSA (Calbiochem-Novabiochem Corp., San Diego, California, USA), once with $\mathrm{Ca}^{2+} / \mathrm{Mg}^{2+}$-free PBS, and then exposed to $500 \mu \mathrm{L}$ dissociation solution (Sigma Chemical Co.) at $37^{\circ} \mathrm{C}$ for 15 minutes. The detached cells were harvested, fixed in $1 \%$ formaldehyde overnight at $4^{\circ} \mathrm{C}$, and incubated with $25 \mu \mathrm{g} / \mathrm{mL}$ of $\mathrm{mAb}$ $2 \mathrm{~F} 23 \mathrm{C} 3$ to $\mathrm{HBP}$ in PBS, including $0.02 \% \mathrm{NaN}_{3}$ and $1 \%$ heat-inactivated human serum, in the presence or absence of 1\% saponin and $0.0125 \%$ digitonin (Sigma Chemical Co.). Next, the cells were incubated with FITCconjugated goat anti-mouse Ig diluted 1:100 in the same buffer. The cells $\left(5 \times 10^{3}\right.$ per experiment) were analyzed on a FACSort (Becton Dickinson Immunocytometry Systems, San Jose, California, USA) using a FACStation with CellQuest software. Background staining was the same in the absence of either HBP or primary antibody. The fluorescence intensity was calculated on channel values and normalized (see Figure 2). The results are given as 
means \pm SD. In some experiments, cells were treated for 1 hour with $50 \mathrm{mM} \mathrm{NH}_{4} \mathrm{Cl}, 1 \mu \mathrm{M}$ cytochalasin $\mathrm{D}$, or 1 $\mathrm{nM}$ cycloheximide prior to and following incubation with HBP for 30 minutes. In another experiment, a mixture of $50 \mu \mathrm{g} / \mathrm{mL}$ of HBP and $100 \mu \mathrm{g} / \mathrm{mL}$ heparin was prepared for 30 minutes before addition to the cells.

Confocal laser microscopy. HUVECs grown overnight on microscope slides were incubated with $50 \mu \mathrm{g} / \mathrm{mL}$ FITClabeled or unconjugated HBP in M199/HBSS for various periods of time. After a brief wash with PBS, the cells were fixed in $4 \%$ formaldehyde for 1 hour. Cells were washed with $100 \mathrm{mM}$ glycine for 1 hour, permeabilized with cold methanol for 10 minutes, and incubated with $1 \%$ BSA in PBS before incubation with 10 $\mu \mathrm{g} / \mathrm{mL}$ of anti-p33 for 30 minutes, followed by 30 $\mu \mathrm{g} / \mathrm{mL}$ Texas red-conjugated goat anti-rabbit Ig. The slides were equilibrated and mounted with SlowFade Antifade (Molecular Probes Europe BV, Leiden, the Netherlands) following the manufacturer's instructions. Cells were viewed at $590 \mathrm{~nm}$, with excitation at $543 \mathrm{~nm}$, using an LSM 310 Laser Scan microscope (Carl Zeiss, Oberkochen, Germany). Horizontal sections of double-stained cells were used for colocalization studies; for FITC, the excitation wavelength was at $488 \mathrm{~nm}$ and the emission was at $525 \mathrm{~nm}$. A color shift from green and red to yellow was considered indicative of colocalization; alternatively, the subtraction method was applied where black areas indicated colocalization. When unconjugated HBP was used, the fixed cells were incubated with $50 \mu \mathrm{g} / \mathrm{mL}$ of biotinylated rabbit antiHBP for 30 minutes, followed by $10 \mu \mathrm{g} / \mathrm{mL}$ of FITCconjugated streptavidin for 30 minutes.
Electron microscopy. HUVECs were grown on polycarbonate filters (Corning-Costar Corp., Cambridge, Massachusetts, USA) with a pore size of $3 \mu \mathrm{m}$. Cells were washed twice in M199/HBSS and incubated for 3 hours in $50 \mu \mathrm{g} / \mathrm{mL}$ HBP added to the upper and lower compartments. Cells were fixed in $0.5 \%$ glutaraldehyde and $1.5 \%$ paraformaldehyde in $0.1 \mathrm{M}$ Sörensen buffer at $\mathrm{pH} 7.3$ for 1 hour, dehydrated in ethanol, and then further processed for Lowicryl embedding (33). For immunostaining, free aldehyde groups were blocked with $50 \mathrm{mM}$ glycine and incubated for 1 hour with 5\% Aurion BSA (Aurion ImmunoGold Reagents and Accesories, Wageningen, the Netherlands) and $5 \%$ goat sera in PBS. Next, the sections were incubated with $0.2 \%$ Aurion BSA, $0.1 \%$ fish gelatin (Amersham International) in PBS for $3 \times 15$ minutes, followed by overnight incubation with $5 \mu \mathrm{g} / \mathrm{mL}$ rabbit anti-human $\mathrm{HBP}$ diluted in the same buffer. Sections were washed in $0.1 \%$ Tween-20 followed by PBS, and incubated with 15-nm gold-labeled goat anti-rabbit antibodies diluted 1:20. After washes (described above), the sections were postfixed in $2 \%$ glutaraldehyde. The sections were further processed overnight with mAb 1273 at 1:50, followed by $10-\mathrm{nm}$ gold-labeled goat anti-mouse antibody at 1:20. Sections were contrast-stained with uranyl acetate and viewed in an electron microscope.

HBP internalization. The internalization rate constant $\left(K_{\mathrm{I}}\right)$ of ${ }^{125}$ I-labeled HBP was determined by the method of Wiley and Cunningham (34). Briefly, HUVECs were incubated with $0.65 \mathrm{nM}{ }^{125} \mathrm{I}$-labeled HBP at $4^{\circ} \mathrm{C}$ or $37^{\circ} \mathrm{C}$ in $0.25 \mathrm{~mL} \mathrm{M199}$. At indicated times, the cells were washed with $1.0 \mathrm{~mL} \mathrm{M199}$ and treated with 0.25 a

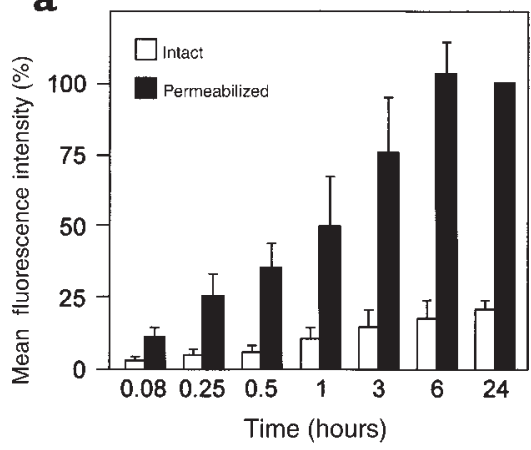

b

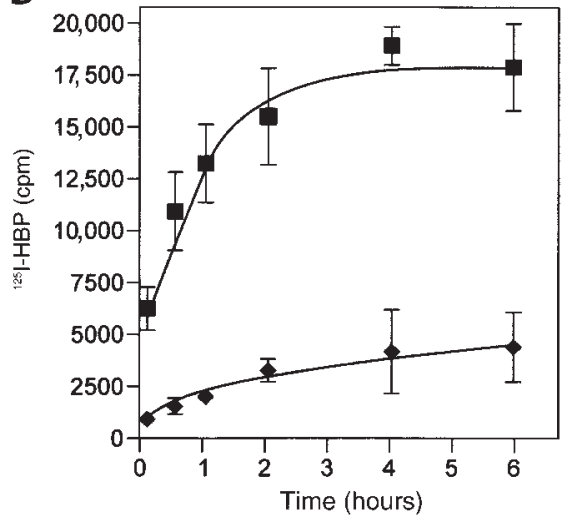

c

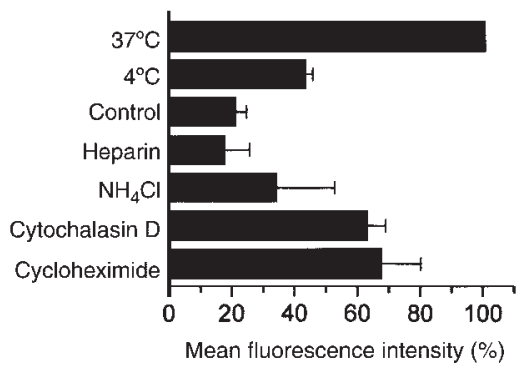

Figure 2

Internalization of HBP by HUVECs. (a) HUVECs were incubated with $50 \mu \mathrm{g} / \mathrm{mL}$ of HBP for the indicated periods of time. The cells were washed, fixed in 1\% formaldehyde, permeabilized, and stained with mAb 2F23C3 to human HBP, followed by an FITC-labeled anti-mouse Ig (from goat); they were then subjected to flow cytometry. Negative controls were stained excluding the primary antibody. Background staining was subtracted. The data represent mean fluorescence intensity normalized for HBP binding at 24 hours and are given as means \pm SD. (b) HUVECs were incubated with $0.65 \mathrm{nM}$ of ${ }^{125}$-labeled HBP at $37^{\circ} \mathrm{C}$. At the indicated times, the radioactivity present on the cell surface (squares) or in the interior of the cell (diamonds) was quantified. Data represent means \pm SD of 3 independent experiments, each done in duplicate. (c) HUVECs were incubated with $50 \mu \mathrm{g} / \mathrm{mL} \mathrm{HBP}$ for 30 minutes at $37^{\circ} \mathrm{C}$, washed, fixed, permeabilized, incubated with mAb 2F23C3 and FITC-anti-mouse Ig, and analyzed by flow cytometry (top bar). To test for specificity, cells were incubated with buffer alone (Control) at $4^{\circ} \mathrm{C}$ or with a mixture of $50 \mu \mathrm{g} / \mathrm{mL} \mathrm{HBP}$ and $100 \mu \mathrm{g} / \mathrm{mL}$ heparin. Alternatively, cells were incubated for 60 minutes with $50 \mathrm{mM}$ $\mathrm{NH}_{4} \mathrm{Cl}, 1 \mathrm{nM}$ cycloheximide, or $1 \mu \mathrm{M}$ cytochalasin D before adding $50 \mu \mathrm{g} / \mathrm{mL} \mathrm{HBP}$. Data represent mean fluorescence intensity normalized for $\mathrm{HBP}$ binding at $37^{\circ} \mathrm{C}(=100 \%)$ and are given as means $\pm \mathrm{SD}(n=5)$. 
$\mathrm{mL} 0.1 \mathrm{M}$ sodium acetate, $0.1 \mathrm{M} \mathrm{NaCl}(\mathrm{pH} 2.5)$ for 5 minutes (surface bound fraction [Sur]). Then cells were lysed with $0.25 \mathrm{~mL} 0.1 \mathrm{M} \mathrm{NaOH}, 1 \%$ (wt/vol) SDS (internalized fraction [In]). The radioactivity associated with surface-bound fraction or the internalized fraction was quantified, and the internalization rate constant was calculated as $d(\mathrm{In} / \mathrm{Sur}) / d \mathrm{t}$.

Effect of HBP on HUVEC apoptosis. HUVECs in their first passage were trypsinized, seeded at a density of $10^{4}$ cells per well in 96-well culture plates (Packard Instrument Co., Meriden, Connecticut, USA), precoated with gelatin, and grown to confluence in complete growth medium as described. After overnight culture, the medium was changed to M199/Earle's salt, including 10\% FCS (GIBCO BRL). HBP was added to final concentrations of 0,10 , or $50 \mu \mathrm{g} / \mathrm{mL}$, and cells were incubated for 24 hours. Apoptosis was induced by replacing the HBP-containing medium with M199/Earle's salt in the absence of FCS. Alternatively, cells were kept in M199/Earle's salt with $10 \%$ FCS. After 24 hours of incubation, DNA fragmentation was measured by the TUNEL method (35) using terminal deoxynucleotidyl transferase and $\left.{ }^{[3} \mathrm{P}\right] \mathrm{dCTP}$ (Amersham International). For control, cells were incubated without transferase under otherwise identical conditions. Specific labeling was obtained by subtracting the background level of the controls. To follow the caspase-3 activation, HUVECs were cultured and seeded at a density of 200,000 cells per well in 6-well plates as detailed earlier here. HBP was added to final concentrations of $0,1,5,10$, or $50 \mu \mathrm{g} / \mathrm{mL}$, incubated for 24 hours, and apoptosis was induced as described. Caspase-3 activity was measured by the CaspACE Assay System (Promega Corp.) following the instructions of the manufacturer. Released 7-amino-4-methyl coumarin (AMC) was detected by a fluorescence plate reader using excitation and emission filters at 355 and $460 \mathrm{~nm}$, respectively. In parallel runs, the caspase- 3 inhibitor AcDEVD-CHO was added; the resultant values were regarded as background and subtracted from the experimental values. A calibration curve was established using an AMC standard to calculate the amount of cleaved DEVD-AMC substrate (pmol AMC/Mg protein).

\section{Results}

Release of HBP from neutrophils and binding of HBP to HUVECs. HBP is a protein that is synthesized and stored in PMNs (5). To demonstrate that HBP can be released from these cells, we stimulated human neutrophils with increasing concentrations of PMA, FMLP, or leukotriene $\mathrm{B}_{4}$ in the presence or absence of HUVECs. The agonisttriggered release of HBP was followed by sandwich ELISA and is exemplified for PMA (Figure 1a). PMA and FMLP promoted HBP release from neutrophils in a dose-dependent manner, whereas leukotriene $\mathrm{B}_{4}$ failed to cause HBP secretion (data not shown). The presence of endothelial cells tended to further increase the release of neutrophil-borne HBP. Hence, stimulated human neutrophils have the capacity to efficiently secrete HBP in proximity to HUVECs. We wondered what the fate of
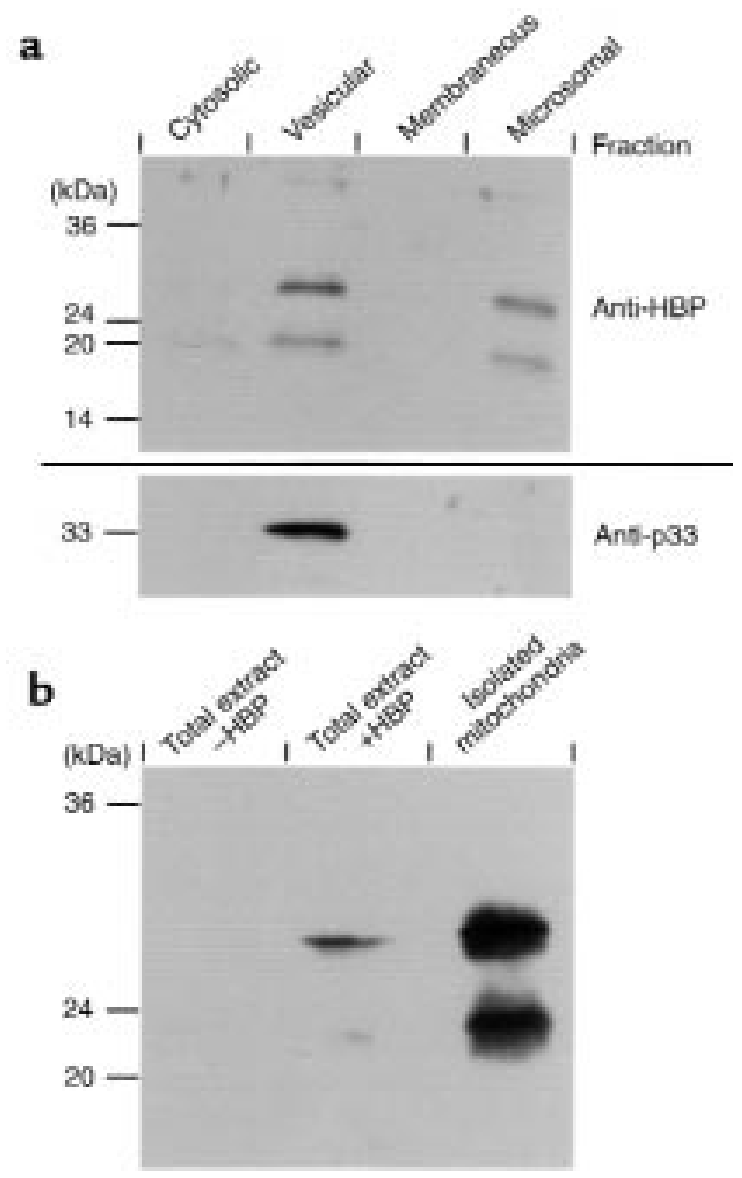

Figure 3

Immunoblotting of HBP and p33 in subcellular fractions and isolated mitochondria of HUVECs. (a) Proteins from the cytosolic fraction (S3), vesicular fraction (P3), membrane fraction (P4), and microsomal fraction (P5) were separated by SDS-PAGE, blotted to nitrocellulose, and detected with polyclonal anti-HBP (top) or anti-p33 (bottom). The nomenclature given (S3, P3, P4, P5) follows the form used previously (21). (b) Total extracts from HUVECs preincubated in the absence or presence of $50 \mu \mathrm{g} / \mathrm{mL}$ HBP for 12 hours, or isolated mitochondria of HBP-treated cells, were applied to SDS-PAGE ( $30 \mu \mathrm{g}$ of protein per lane), blotted to nitrocellulose, and detected with anti-HBP. Relative molecular masses of marker proteins are given on the left.

the released HBP might be. Because HBP binds with high affinity to heparin (15), our initial hypothesis was that neutrophil-borne HBP may interact with endothelial cells through heparin-like surface molecules such as heparan sulfate-containing proteoglycans. We metabolically labeled HUVECs with ${ }^{35} \mathrm{~S}_{\mathrm{SO}_{4}-}{ }^{2-}$ to allow incorporation into sulfated carbohydrate structures. After 24 hours of incubation, the cells were lysed, and the total cellular lysate was affinity-purified on HBP-streptavidin agarose. SDS-PAGE of the radioactive material eluted from the HBP column gave a broad smear of bands over a molecular mass range of $40 \mathrm{kDa}$ to more than $400 \mathrm{kDa}$ (data not shown), as typically observed for proteoglycans (22). To characterize further the affinity-purified material, we employed enzymatic and chemical degradation of the radiolabeled products. Eluted material from the $\mathrm{HBP}$ column was treated with $\mathrm{C}_{\mathrm{ABC}}$ or $\mathrm{HNO}_{2}$. The result- 


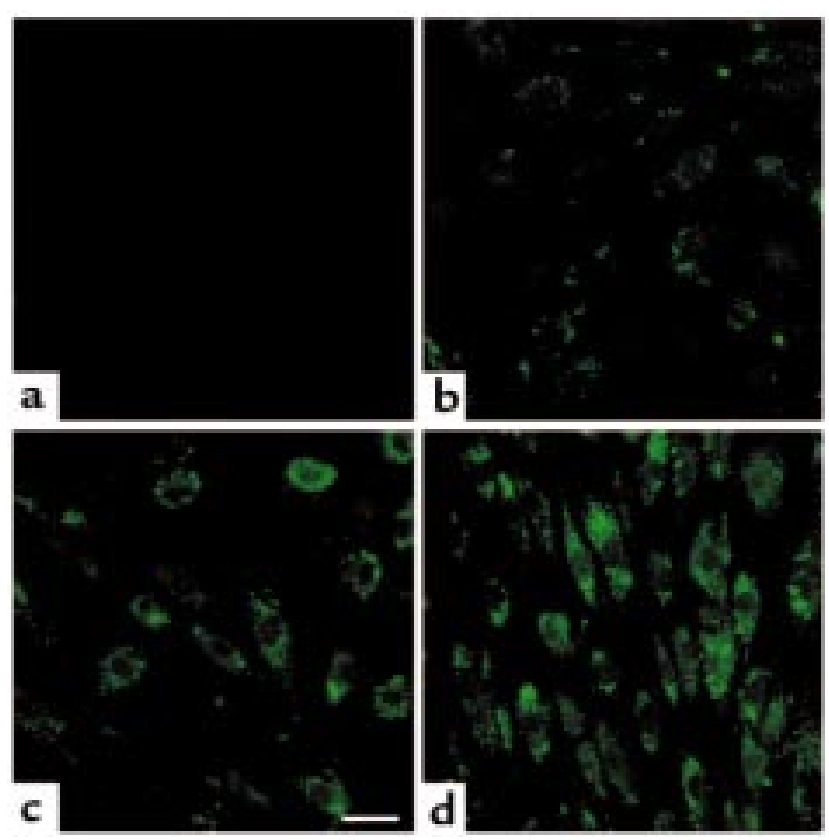

Figure 4

Time course of internalization and subcellular distribution of HBP. For control, the biotinylated antibody was omitted (a). HUVECs were incubated with $50 \mu \mathrm{g} / \mathrm{mL} \mathrm{HBP}$ for 0.5 hours (b), 2 hours (c), and 24 hours (d). Cells were washed to remove unbound ligand, fixed with $4 \%$ formaldehyde, and successively incubated with $50 \mu \mathrm{g} / \mathrm{mL}$ biotinylated anti-HBP and $10 \mu \mathrm{g} / \mathrm{mL}$ FITC-conjugated streptavidin. Internalized HBP was viewed using a confocal laser scanning microscope at an excitation wavelength of $488 \mathrm{~nm}$. $\times 300$. Scale bar: $4 \mu \mathrm{m}$.

ing mixtures were electrophoretically separated on agarose gels (Figure 1b). The ${ }^{35} \mathrm{~S}$-labeled material was sensitive to treatment with $\mathrm{C}_{\mathrm{ABC}}$ (top) or $\mathrm{HNO}_{2}$ (bottom), indicating that proteoglycans containing chondroitin sulfate and dermatan sulfate side chains sensitive to $\mathrm{C}_{\mathrm{ABC}}$, and/or heparan sulfate side chains sensitive to $\mathrm{HNO}_{2}$, were bound by immobilized HBP. No radioactivity in the position of the free glycosaminoglycan chains were found after $\mathrm{HNO}_{2}$ treatment (Figure 1b). Treatment with heparinase III, which cleaves heparan sulfate side chains, partially digested HBP-binding proteoglycans, and the combined action of heparinase III and $\mathrm{C}_{\mathrm{ABC}}$ completely degraded them (data not shown).

Identification of HBP-binding proteoglycans. Endothelial cells are known to express proteoglycans such as perlecan, glypican, syndecan-1, $-2,-3$, and -4 of the heparan sulfate type, and biglycan and decorin of the chondroitin sulfate type. We digested affinity-purified HBP binding sites with a combination of heparitinase and $\mathrm{C}_{\mathrm{ABC}}$ to completely remove their glycosaminoglycan side chains. The resulting cleavage products were separated by SDS-PAGE and subjected to Western blotting using mAb 3G10 (22), which selectively binds to a neoepitope (i.e., desaturated glucuronate) that is generated upon heparitinase treatment of heparan sulfate-type proteoglycans (Figure 1c). For comparison, proteoglycans of total HUVEC lysates were run in parallel and identified by the relative molecular masses of their core proteins. The heparan sulfate-containing proteoglycans present in total HUVEC lysates were also present in the HBP-binding fraction, thus proving that proteoglycans represent major docking sites for HBP. The binding of HBP was not restricted to a particular proteoglycan present in the HUVEC extract, consistent with the notion that HBP binds to the glycosaminoglycan moieties rather than to the core proteins of the proteoglycans associated with endothelial cells.

Internalization of HBP. Because syndecans have been shown to mediate internalization of external proteins (36), we asked whether binding of HBP to proteoglycans may be followed by its uptake by HUVECs. To address this hypothesis, we incubated unlabeled HBP with cultured HUVECs for various time periods. After extensive washing, the cells were fixed, permeabilized, and incubated with the mAb to HBP, followed by an FITC-conjugated secondary antibody. Flow cytometry revealed an intracellular accumulation of HBP that steadily increased over the first 6 hours and then plateaued (Figure 2a). Control experiments, where HBP or the first antibody had been omitted, demonstrated the specificity of the test system (not shown). Unlike permeabilized cells, the intact HUVECs showed only a minor increase of fluorescence intensity (Figure 2a), indicating that a significant fraction of the ligand had been sequestered from the cell surface before fixation. We conclude that HUVECs bind and internalize exogenous HBP. To address further the role of proteoglycans in cellular HBP internalization, we employed a variant of $\mathrm{CHO}$ cells (pgsD-677) that is deficient in heparan sulfate proteoglycans. Heparan sulfate-deficient pgsD-677 cells internalized HBP with reduced efficiency (data not shown), suggesting that sites other than heparan sulfate-type proteoglycans capable of binding and sequestering HBP must exist, e.g., chondroitin sulfate-containing proteoglycans overexpressed by the pgsD-677 cells (27). These data also demonstrate that cells other than HUVECs specifically bind and internalize HBP, suggesting that we are not dealing with a cell type-specific phenomenon.

Characterization of HBP internalization. To quantify the internalization process, we radiolabeled human HBP with ${ }^{125} \mathrm{I}$ and followed the kinetics of the HBP uptake by HUVECs. The internalization rate constant $K_{\mathrm{I}}$ for radiolabeled HBP was $0.035 \pm 0.0091 \mathrm{~min}^{-1}$ at $37^{\circ} \mathrm{C}$ (Figure $2 \mathrm{~b})$. This process was reversible, because increasing concentrations of unlabeled HBP effectively competed for internalization with an apparent $\mathrm{IC}_{50}$ of $78.3 \pm 23.8 \mathrm{nM}$. Unexpectedly, we also observed internalization at $4^{\circ} \mathrm{C}$, though at a considerably lower rate of $K_{\mathrm{I}}=0.008 \pm$ $0.0065 \mathrm{~min}^{-1}$ (see below). To study the HBP internalization process in more detail, we performed flow cytometry of HUVECs preincubated with $50 \mu \mathrm{g} / \mathrm{mL}$ of HBP at $37^{\circ} \mathrm{C}$ for 0.5 hours, followed by extensive washing to remove unbound ligand (Figure $2 \mathrm{c}$ ). As a negative control, cells were incubated in the absence of HBP. Preincubation of HBP with $100 \mu \mathrm{g} / \mathrm{mL}$ heparin effectively prevented uptake of the ligand by $83 \pm 8 \%$, most likely owing to competition with glycosaminoglycans for HBP 
binding. Incubation at $4{ }^{\circ} \mathrm{C}$ attenuated HBP internalization by $53 \pm 3 \%$, and addition of $\mathrm{NH}_{4} \mathrm{Cl}$ reduced $\mathrm{HBP}$ internalization by $66 \pm 19 \%$. Cytochalasin D, an inhibitor of actin filament polymerization, lowered HBP internalization by $37 \pm 6 \%$, whereas cycloheximide, an inhibitor of protein synthesis, reduced HBP internalization by 32 $\pm 12 \%$. Collectively, these findings indicate that HBP internalization is a receptor-mediated process that requires an intact and functional cytoskeleton.

Subcellular fractionation of HBP-treated HUVECs. What are the intracellular target compartments of HBP? To address this issue, we used cell fractionation to examine the subcellular localization of HBP in HUVECs that had been incubated with unlabeled HBP for 24 hours at $37^{\circ} \mathrm{C}$ before lysis. Equal amounts of protein from the various cell fractions were subjected to Western blotting using anti-HBP (Figure 3a, top). A major 28$\mathrm{kDa}$ band and a minor $22-\mathrm{kDa}$ band were present in the vesicular and microsomal fractions, but not in the cytosolic or membrane fractions. The majority of internalized HBP retained the molecular mass of the native protein of $28 \mathrm{kDa}$, suggesting that it is still present in the full-length form. HUVECs that had been kept with buffer alone failed to reveal specific immunoreactive bands (not shown), confirming that native HUVECs do not express appreciable amounts of HBP. An endogenous marker protein, $\mathrm{p} 33$, present in the vesicular fraction of HUVECs (21), verified the efficiency of the fractionation procedure (Figure 3a, bottom). We conclude that HBP is taken up by HUVECs and routed to their vesicular and/or microsomal compartments.

Subcellular localization of internalized HBP. To define more precisely the intracellular targets of internalized HBP, we employed confocal laser microscopy. When unlabeled HBP was applied to HUVECs, followed by a biotinylated antibody to HBP and FITC-conjugated streptavidin, a specific granular staining pattern of $\mathrm{HBP}$ in HUVECs became apparent (Figure 4). Controls in the absence of anti-HBP (Figure 4a), or in the presence of preabsorbed anti-HBP (not shown), failed to produce specific staining. The staining intensity increased over time (0.5-24 hours), indicating that HBP is continuously taken up by HUVECs. Staining for HBP was most intense in vesicular structures adjacent to the cell nucleus (Figure 4d). Experiments with FITC-coupled HBP revealed similar staining patterns (data not shown). Because internalized HBP colocalizes with the mitochondrial protein p33 (37) in the vesicular fraction of HUVECs, we performed double staining for the 2 proteins. HUVECs were incubated with FITC-labeled HBP for up to 24 hours, and were fixed and incubated with antibodies to human p33 and Texas red-conjugated anti-rabbit Ig. Controls were done in the absence of FITC-HBP (not shown). Both FITC-conjugated HBP (green) and endogenous p33 antigen (red) were abundant in perinuclear spots (Figure 5, a and b). Confocal overlay of the staining patterns in Figure 5, a and b revealed yellowish spots (Figure 5c). Black spots became visible in the corresponding subtraction overlay (Figure $5 \mathrm{~d}$ ), indicating that the majority of HBP colocalizes with mitochondrial compartments housing p33.

Electron microscopy and Western blotting of isolated mitochondria. To define more precisely the cellular structures associated with HBP, we employed electron microscopy using anti-HBP (15-nm gold particles) and mAb 1273 to a $65-\mathrm{kDa}$ mitochondrial marker protein (10-nm gold particles). Gold particles of both sizes were confined to the same intracellular structures (mitochondria), whereas the nucleus, cytoplasm, and extracellular compartment were essentially free of staining (Figure 6). We observed that cells that had taken up much HBP (as judged by the number of 15 -nm gold particles) had an altered morphology; i.e., their membranes appeared fuzzy. The reasons for this morphological change are unknown. No immunoreactivity for HBP was observed in HUVECs in the absence of exogenous HBP, and no
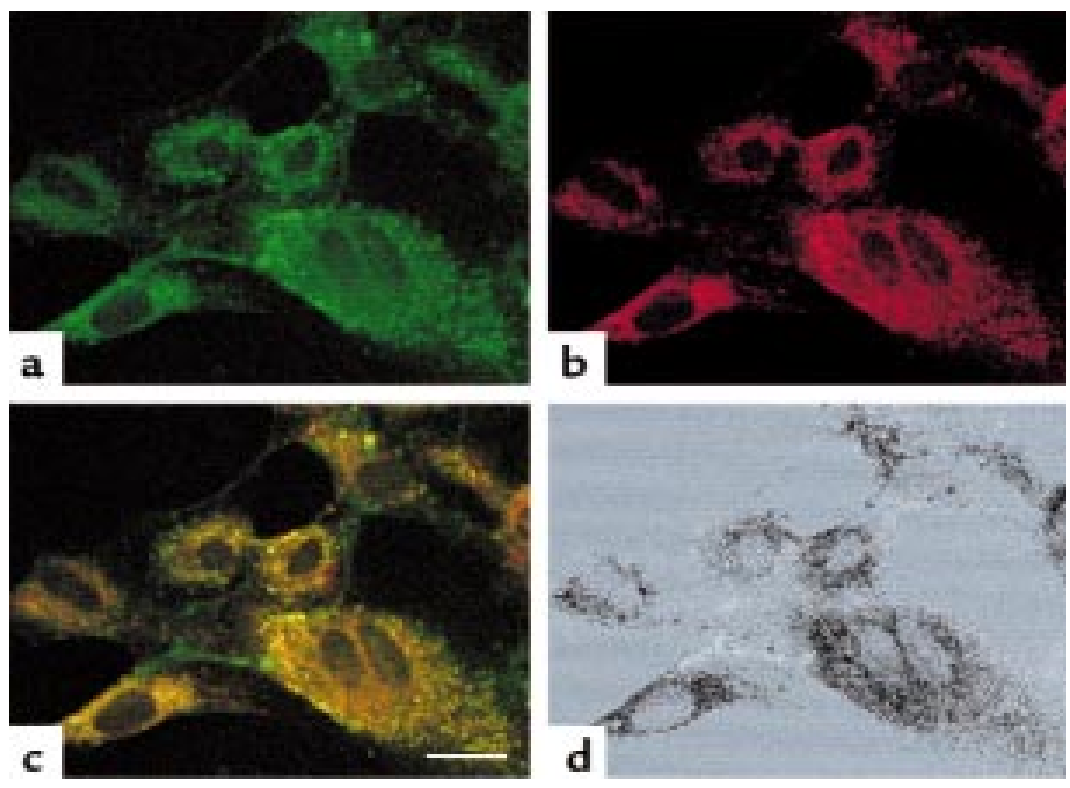

\section{Figure 5}

Colocalization of HBP and p33. HUVECs were incubated with $50 \mu \mathrm{g} / \mathrm{mL}$ FITC-labeled HBP (a) for 6 hours, fixed in $4 \%$ formaldehyde, and incubated with $10 \mu \mathrm{g} / \mathrm{mL}$ anti-p33, followed by $30 \mu \mathrm{g} / \mathrm{mL}$ Texas red-conjugated anti-rabbit Ig (b), and were imaged by confocal laser scanning microscopy. (c) An overlay of $\mathbf{a}$ and $\mathbf{b}$, where yellowish coloring indicates colocalization of HBP and p33. (d) A subtraction overlay of $\mathbf{a}$ and $\mathbf{b}$, where black dots indicate colocalization. $\times 1000$. Scale bar: $25 \mu \mathrm{m}$. The pictures are representative of 3 independent experiments done under identical conditions. 


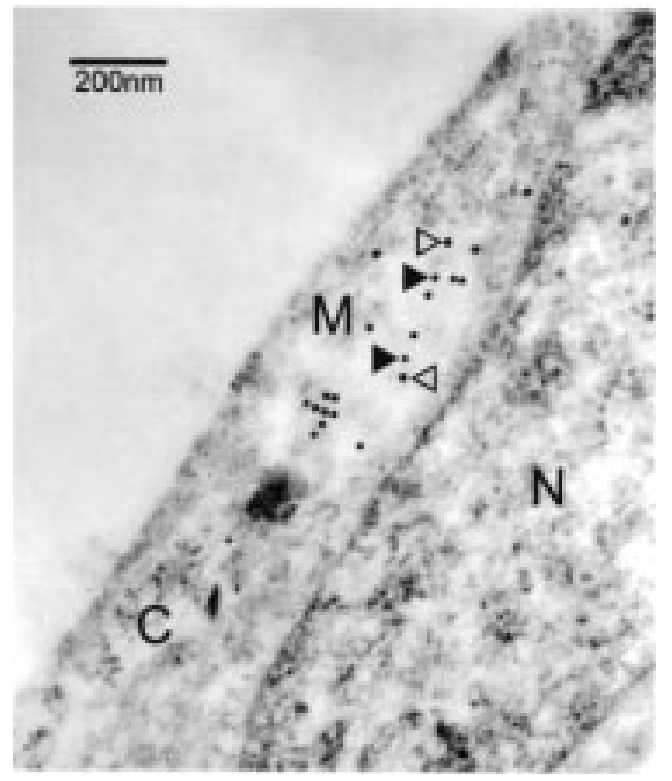

\section{Figure 6}

Electron microscopy of internalized HBP. HUVECs were incubated with $50 \mu \mathrm{g} / \mathrm{mL}$ HBP for 3 hours, and fixed in $0.5 \%$ glutaraldehyde and $1.5 \%$ paraformaldehyde for 1 hour. Staining was done with 5 $\mu \mathrm{g} / \mathrm{mL}$ of rabbit anti-HBP and 15-nm gold-labeled goat anti-rabbit Ig (open arrowheads), and with mouse mAb 1273 (1:50) to a 65$\mathrm{kDa}$ mitochondrial marker protein and $10-\mathrm{nm}$ gold-labeled goat anti-mouse Ig (filled arrowheads). The sections were postfixed in $2 \%$ glutaraldehyde. Scale bar: $200 \mathrm{~nm}$. A representative section is shown. C, cytosol; M, mitochondrion; N, nucleus.

staining was seen with preimmune rabbit sera in sections of HBP-treated cells (data not shown). To probe directly for the association of internalized HBP with mitochondria, we treated HUVECs with $50 \mu \mathrm{g} / \mathrm{mL}$ of HBP for 12 hours and isolated their mitochondria. Western blotting demonstrated the presence of HBP in the total extracts of HBP-treated HUVECs, and a high enrichment of HBP in the mitochondrial fraction of HBP-treated HUVECs (Figure 3b). No HBP was found in untreated HUVECs. Collectively, our findings indicate that a significant fraction of internalized HBP is targeted to the mitochondria of endothelial cells.

Functional role of internalized HBP in endothelial cell apoptosis. Given the fact that internalized HBP is in association with mitochondrial compartments, we wondered whether HBP might have a functional role in endothelial cell apoptosis. Therefore, we chose the established model of growth factor deprivation-induced apoptosis to test for potential biological readouts of HBP uptake (38). HUVECs in their second passage were grown to confluence and incubated with varying concentrations (up to $50 \mu \mathrm{g} / \mathrm{mL}$ ) of HBP. After 24 hours, free HBP ligand was removed, and apoptosis was induced by withdrawal of FCS from the medium for the next 24 hours. Cells that had been continuously kept in the presence of FCS-borne growth factors served as the control. DNA fragmentation was measured by the TUNEL assay using terminal deoxynucleotidyl transferase in the presence of $\left[{ }^{33} \mathrm{P}\right] \mathrm{dCTP}$. Extensive $\left[{ }^{33} \mathrm{P}\right] \mathrm{dCTP}$ incorporation, indicating DNA fragmentation, was seen after deprivation in the absence of HBP (Figure 7a). Preincubation of the cells with $10 \mu \mathrm{g} / \mathrm{mL}$ of HBP significantly reduced $\left.{ }^{[3} \mathrm{P}\right] \mathrm{dCTP}$ incorporation into DNA, and treatment with $50 \mu \mathrm{g} / \mathrm{mL}$ of HBP showed a marked reduction of $\left[{ }^{33} \mathrm{P}\right] \mathrm{dCTP}$ incorporation, indicating that HBP effectively prevented apoptosis of HUVECs. This protective effect - though less dramatic - was also seen in control cells kept continuously in the presence of FCS. The cytoprotective effect of HBP was further demonstrated by the quantitative analysis of caspase- 3 activity, indicating a dose-dependent reduction of caspase activation in the presence of HBP (Figure $7 \mathrm{~b}$ ). We observed a significant reduction of caspase-3 activity at $10 \mu \mathrm{g} / \mathrm{mL}$ HBP; at $50 \mu \mathrm{g} / \mathrm{mL}$ of HBP, caspase- 3 activity was down to approximately $50 \%$ of the control in the absence of HBP. Hence, internalization of neutrophil-borne HBP by HUVECs may protect endothelial cells from growth factor deprivationinduced apoptosis.

\section{Discussion}

Proteoglycans bind important proteins involved in inflammatory processes, including bFGF (39) and TGF- $\beta$ (40). Upon binding to cell-surface proteoglycans, these proteins are targeted to high-affinity transmembrane receptors, which, in turn, trigger intracellular signaling cascades $(39,40)$. Thus, proteoglycans serve as carriers and reservoirs for powerful intercellular communicators, act as scavengers to avoid their uncontrolled spreading, and thereby render inflammation a local phenomenon $(39,40)$. Our results indicate that HUVEC surface proteoglycans may serve as primary docking sites for HBP. Major heparan sulfate-type proteoglycans expressed by endothelial cells (i.e., syndecan-1 through syndecan-4, glypican, and perlecan) were enriched by affinity chromatography on immobilized HBP, suggesting that HBP binds to them through their glycosaminoglycan side chains. We have not addressed the structural elements of HBP involved in proteoglycan binding. However, we infer from the distinct surface pattern of charged patches of HBP (13) that ionic interactions between the basic surface area of the protein and the negatively charged glycosaminoglycan side chains of the major endothelial proteoglycans are responsible for these interactions.

It has been shown recently that the syndecan family of proteoglycans provides a new internalization pathway (36). The precise mechanism driving the proteoglycanmediated internalization of protein ligands is not yet understood. Our finding that the heparan sulfate-deficient cell line pgsD-677 (27) showed an attenuated HBP internalization lends further credence to such a proteoglycan-mediated endocytic mechanism. Interestingly, $\mathrm{C}_{A B C}$ treatment of pgsD-677 cells failed to nullify uptake of HBP (data not shown), thus leaving room for the presence of hitherto unknown receptors that could be involved in HBP internalization. Indeed, previous studies have shown that bFGF is internalized by endothelial 
cells (41), and that the internalization of FGF by CHO cells is mediated by cell-surface receptors as well as by heparan sulfate-containing proteoglycans (42). Our present study demonstrates a role for proteoglycans in HBP binding and internalization in vitro, although the observed biological effects of internalized HBP on HUVECs may also point to a function in vivo (see below).

One of the intriguing findings of this study is colocalization of internalized HBP with p33, an established mitochondrial marker protein $(37,43)$. This observation raises questions as to (a) the identity of the compartments targeted by HBP, (b) the intracellular routing of internalized HBP, (c) the integrity of targeted HBP, and (d) the functional roles of internalized HBP. Typical intracellular destinations of endocytosed material are the lysosomes, the Golgi complex, or the cytosol (44). In the case of lysosomal targeting, the position of endocytosed material reaches more central regions of the cell, because early endosomes mature to late endosomes (44). Furthermore, endocytosed material may travel from the Golgi complex to the endoplasmic reticulum (45), and the latter may be found in proximity to mitochondria (46). Indeed, our cell fractionation studies indicate that the microsomal fraction comprising mostly endoplasmic reticulum-derived vesicles contained significant amounts of HBP.

The distinct possibility remains that internalized HBP reaches the cytoplasm and subsequently associates with mitochondria. The results from confocal laser scanning microscopy, electron microscopy, and Western blotting of isolated mitochondria from HBP-treated HUVECs all suggest that a sizeable fraction of neutrophil-borne HBP is targeted through unknown routes to mitochondria, where it may protect endothelial cells against programmed cell death after withdrawal of growth factors (38). This conclusion is supported and extended by our finding that HBP dose dependently reduced caspase- 3 activation. Our present notions are in line with the previous finding that HBP confers improved survival rates and longevity to human monocytes (12). At present, we do not know which of the 2 HBP forms (28 $\mathrm{kDa}$ or $22 \mathrm{kDa}$ ) detected by Western blotting of isolated mitochondria and cell fractions of HBP-treated HUVECs mediates the cytoprotective effects. In flow cytometry we were able to use an mAb directed to an epitope lost upon denaturation. This indicates that a fraction of internalized HBP remains in its intact form.

It has been demonstrated that the $\mathrm{Bcl}-2$ oncoprotein protects cells from apoptosis through yet unknown mechanisms of interaction with the outer mitochondrial membrane (47). Accumulating experimental evidence points to a pivotal role of mitochondria in the initiation of the apoptosis process $(38,48)$. Numerous pathways, including those induced by growth factor withdrawal that drive cells to enter the death pathway, converge on mitochondria. Indeed, formation of mitochondrial megachannels and outflow of cytochrome $c$ from the intermembrane spaces are processes associated with the outer mitochondrial membrane $(38,49)$. It is tempting to speculate that uptake and routing of
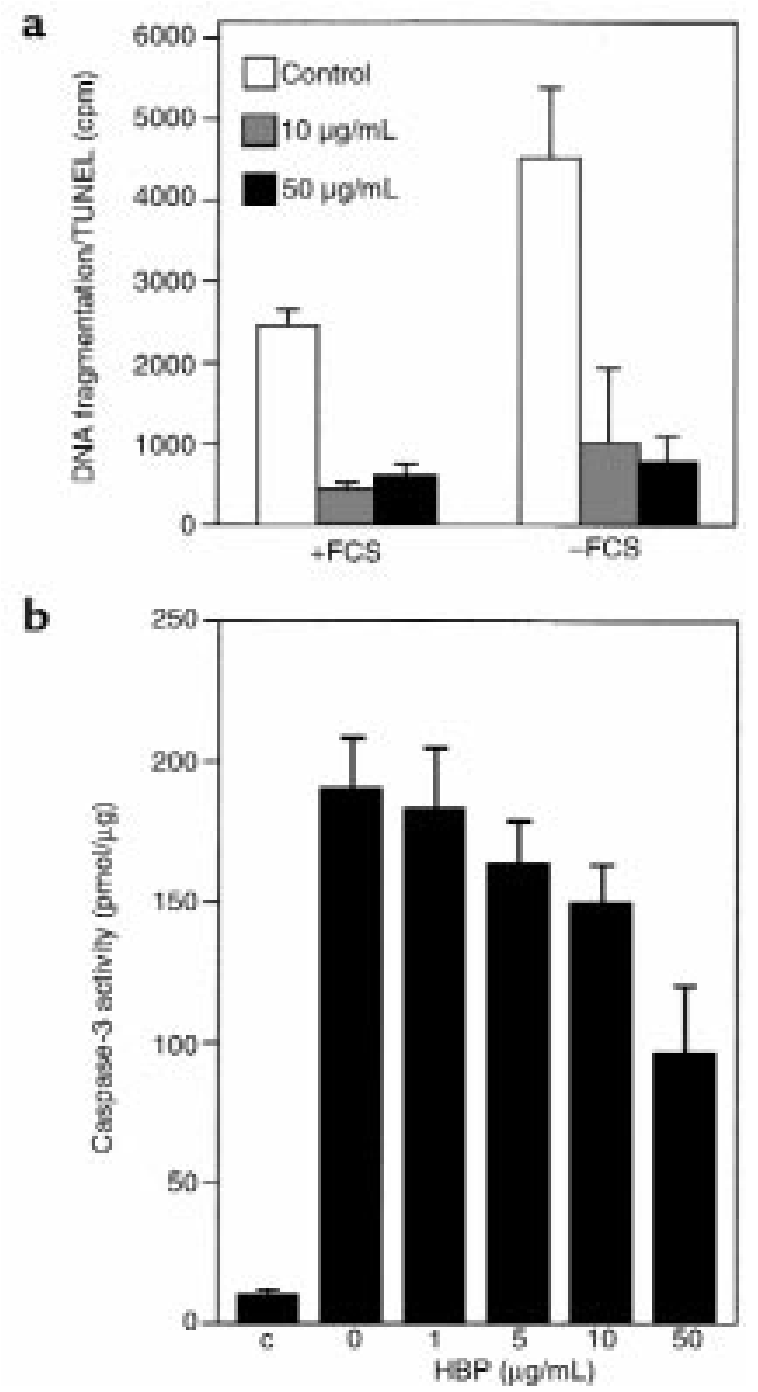

Figure 7

Effect of HBP on endothelial cell apoptosis. (a) HUVECs were incubated for 24 hours with the indicated concentrations of HBP in M199 Earle's salt containing 10\% FCS. The medium was removed, and cells were kept for 18 hours in M199Earle's salt in the presence $(+F C S)$ or absence $(-F C S)$ of $10 \%$ FCS. Controls were processed in the absence of HBP. DNA fragmentation was measured by the TUNEL method and is given as cpm per $10^{4}$ cells. Values represent means \pm SD $(n=3)$. (b) Inhibition of caspase- 3 activation by HBP. HUVECs were incubated with increasing concentrations of HBP $(0-50 \mu \mathrm{g} / \mathrm{mL})$ for 24 hours. Then cells were incubated in FCS-free M199Earle's salt medium, and caspase- 3 activity was measured after 18 hours. For control (c), cells were kept in the continuous presence of $10 \%$ FCS without previous HBP addition. Activity is presented as picomole of AMC liberated per microgram of protein.

HBP to mitochondria is a prerequisite for its protective role against apoptosis in endothelial cells. Thus, HBP may serve important roles as an intercellular signal connecting neutrophils and endothelial cells, and may help to minimize endothelial cell damage in the context of circumscribed neutrophil activation in vivo. More detailed studies are warranted to unravel the molecular and cellular mechanisms underlying the delicate role of neutrophil-borne HBP in endothelial cells. 


\section{Acknowledgments}

This work was supported by grants from the Swedish Society for Medical Research, Greta och Johan Kocks Stiftelse, Alfred Österlunds Stiftelse. The authors wish to thank Christel Wängnerud, Andrea Kontros, Lone Hansen, Sanni Rasmussen, and Monica Heidenholm for technical assistance; Michael Plenikowski and Birgitta Jönsson for artwork; and Matthias Mörgelin, Eric Carlemalm, and Lina Gefors for experimental help.

1. McIntyre, T.M., Modur, V., Prescott, S.M., and Zimmerman, G.A. 1997. Molecular mechanisms of early inflammation. Thromb. Haemost. 78:302-305.

2. Thelen, M., Dewald, B., and Baggiolini, M. 1993. Neutrophil signal transduction and activation of the respiratory burst. Physiol. Rev. 73:797-821.

3. Carlos, T.M., and Harlan, J.M. 1994. Leukocyte-endothelial adhesion molecules. Blood. 84:2068-2101.

4. Borregaard, N., et al. 1993. Human neutrophil granules and secretory vesicles. Eur. J. Haematol. 51:187-198.

5. Pereira, H.A. 1995. CAP37, a neutrophil-derived multifunctional inflammatory mediator. J. Leukoc. Biol. 57:805-812.

6. Gabay, J.E., et al. 1989. Antibiotic proteins of human polymorphonuclear leukocytes. Proc. Natl. Acad. Sci. USA. 86:5610-5614.

7. Pereira, H.A., Erdem, I., Pohl, J., and Spitznagel, J.K. 1993. Synthetic bactericidal peptide based on CAP37: a 37-kDa human neutrophil granuleassociated cationic antimicrobial protein chemotactic for monocytes. Proc. Natl. Acad. Sci. USA. 90:4733-4737.

8. Mercer-Jones, M.A., Peyton, J., Cook, M., Flodgaard, H., and Cheadle, W.G. 1996. Monocyte recruitment increase survival in fecal peritonitis. Surg. Forum. 37:105-108.

9. Pereira, H.A., Shafer, W.M., Pohl, J., Martin, L.E., and Spitznagel, J.K 1990. CAP37, a human neutrophil-derived chemotactic factor with monocyte specific activity. J. Clin. Invest. 85:1468-1476.

10. Rasmussen, P.B., et al. 1996. Characterization of recombinant human $\mathrm{HBP} / \mathrm{CAP} 37 /$ azurocidin, a pleiotropic mediator of inflammationenhancing LPS-induced cytokine release from monocytes. FEBS Lett. 390:109-112

11. Chertov, O., et al. 1996. Identification of defensin-1, defensin-2, and CAP37/azurocidin as T-cell chemoattractant proteins released from interleukin-8-stimulated neutrophils. J. Biol. Chem. 271:2935-2940.

12. Ostergaard, E., and Flodgaard, H. 1992. A neutrophil-derived proteolytic inactive elastase homologue (hHBP) mediates reversible contraction of fibroblasts and endothelial cell monolayers and stimulates monocyte survival and thrombospondin secretion. J. Leukoc. Biol. 51:316-323.

13. Iversen, L.F., et al. 1997. Structure of HBP, a multifunctional protein with a serine proteinase fold. Nat. Struct. Biol. 4:265-268.

14. Almeida, R.P., Melchior, M., Campanelli, D., Nathan, C., and Gabay, J.E. 1991. Complementary DNA sequence of human neutrophil azurocidin, an antibiotic with extensive homology to serine proteases. Biochem. Biophys. Res. Commun. 177:688-695.

15. Flodgaard, H., et al. 1991. Covalent structure of two novel neutrophile leucocyte-derived proteins of porcine and human origin. Neutrophil elastase homologues with strong monocyte and fibroblast chemotactic activities. Eur. J. Biochem. 197:535-547.

16. Morgan, J.G., et al. 1991. Cloning of the cDNA for the serine protease homolog CAP37/azurocidin, a microbicidal and chemotactic protein from human granulocytes. J. Immunol. 147:3210-3214.

17. Hofmann, K., Titus, G., Montibeller, J.A., and Finn, F.M. 1982. Avidin binding of carboxyl-substituted biotin and analogues. Biochemistry. 21:978-984.

18. Rinderknecht, H. 1962. Ultra-rapid fluorescent labelling of proteins. Nature. 93:167-168

19. Bolton, A.E., and Hunter, W.M. 1973. The labelling of proteins to high specific radioactivities by conjugation to a ${ }^{125}$ I-containing acylating agent. Biochem. J. 133:529-539.

20. Lindmark, A., Flodgaard, H., and Gullberg, U. 1999. Processing of the neutrophil granule serine protease homologue azurocidin. J. Leukoc. Biol. In press.

21. Dedio, J., and Müller-Esterl, W. 1996. Kininogen binding protein p33/gC1qR is localized in the vesicular fraction of endothelial cells. FEBS Lett. 399:255-258.

22. David, G., Bai, X.M., Van der Schueren, B., Cassiman, J.J., and Van den
Berghe, H. 1992. Developmental changes in heparan sulfate expression: in situ detection with mAbs. J. Cell Biol. 119:961-975.

23. Lewis, J.M., et al. 1991. Subcellular distribution of the $\alpha$ subunit(s) of $G_{i}$ : visualization by immunofluorescent and immunogold labeling. Cell Regul. 2:1097-1113.

24. Hjorth, R., Jonsson, A.K., and Vretblad, P. 1981. A rapid method for purification of human granulocytes using percoll. A comparison with dextran sedimentation. J. Immunol. Methods. 43:95-101.

25. Jaffe, E.A., Nachman, R.L., Becker, C.G., and Minick, C.R. 1973. Culture of human endothelial cells derived from umbilical veins. Identification by morphologic and immunologic criteria. J. Clin. Invest. 52:2745-2756.

26. Thornton, S.C., Mueller, S.N., and Levine, E.M. 1983. Human endothelial cells: use of heparin in cloning and long-term serial cultivation. Science. 222:623-625.

27. Murphy-Ullrich, J.E., Westrick, L.G., Esko, J.D., and Mosher, D.F. 1988. Altered metabolism of thrombospondin by Chinese hamster ovary cells defective in glycosaminoglycan synthesis. J. Biol. Chem. 263:6400-6406.

28. Rickwood, D., Wilson, M.T., and Darley-Usmar, V.M. 1987. Isolation and characteristics of intact mitochondria. In Mitochondria. V.M. DarleyUsmar, editor. IRL Press. Oxford, United Kingdom. 1-6.

29. Laemmli, U.K. 1970. Cleavage of structural proteins during the assembly of the head of bacteriophage T4. Nature. 227:680-685.

30. Björnsson, S. 1993. Simultaneous preparation and quantitation of proteoglycans by precipitation with alcian blue. Anal. Biochem. 210:282-291.

31. Shively, J.E., and Conrad, H.E. 1976. Formation of anhydrosugars in the chemical depolymerization of heparin. Biochemistry. 15:3932-3942.

32. Björnsson, S. 1993. Size-dependent separation of proteoglycans by electrophoresis in gels of pure agarose. Anal. Biochem. 210:292-298.

33. Carlemalm, E., and Villiger, W. 1989. Low temperature embedding. In Techniques in immunocytochemistry. Volume 4. G.R. Bullock and P. Petrusz, editors. Academic Press. London, United Kingdom. 29-45.

34. Wiley, H.S., and Cunningham, D.D. 1982. The endocytotic rate constant. A cellular parameter for quantitating receptor-mediated endocytosis. $J$. Biol. Chem. 257:4222-4229.

35. Gorczyca, W., Gong, J., Ardelt, B., Traganos, F., and Darzynkiewicz, Z. 1993. The cell cycle-related differences in the susceptibility of HL-60 cells to apoptosis induced by various antitumor agents. Cancer Res. 53:3186-3192.

36. Fuki, I.V., et al. 1997. The syndecan family of proteoglycans. Novel receptors mediating internalization of atherogenic lipoproteins in vitro. $J$. Clin. Invest. 100:1611-1622.

37. Dedio, J., Jahnen-Dechent, W., Bachmann, M., and Müller-Esterl, W. 1998. The multiligand-binding protein $\mathrm{gC} 1 \mathrm{qR}$, putative $\mathrm{C} 1 \mathrm{q}$ receptor, is a mitochondrial protein. J. Immunol. 160:3534-3542.

38. Santos, A., Zamtami, N., and Kroemer, G. 1998. Mitochondria as regulators of apoptosis: doubt no more. Biochim. Biophys. Acta. 1366:151-165.

39. Galzie, Z., Kinsella, A.R., and Smith, J.A. 1997. Fibroblast growth factors and their receptors. Biochem. Cell Biol. 75:669-685.

40. Derynck, R., and Feng, X.H. 1997. TGF- $\beta$ receptor signaling. Biochim. Biophys. Acta. 1333:105-150.

41. Gleizes, P.E., Noaillac-Depeyre, J., Amalric, F., and Gas, N. 1995. Basic fibroblast growth factor (FGF-2) internalization through the heparan sulfate proteoglycans-mediated pathway: an ultrastructural approach. Eur. J. Cell Biol. 66:47-59.

42. Roghani, M., and Moscatelli, D. 1992. Basic fibroblast growth factor is internalized through both receptor-mediated and heparan sulfate-mediated mechanisms. J. Biol. Chem. 267:22156-22162.

43. Muta, T., et al. 1997. p32 protein, a splicing factor 2 -associated protein, is localized in mitochondrial matrix and is functionally important in maintaining oxidative phosphorylation. J. Biol. Chem. 272:24363-24370.

44. Mukherjee, S., Ghosh, R.N., and Maxfield, F.R. 1997. Endocytosis. Physiol. Rev. 77:759-803.

45. Lord, J.M., and Roberts, L.M. 1998. Retrograde transport: going against the flow. Curr. Biol. 8:R56-R58

46. Rizzuto, R., et al. 1998. Close contacts with the endoplasmic reticulum as determinants of mitochondrial $\mathrm{Ca}^{2+}$ responses. Science. 280:1763-1766.

47. Zamzami, N., Brenner, C., Marzod, I., Susin, S.A., and Kroemer, G. 1998. Subcellular and submitochondrial mode of action of the Bcl2-linked oncoproteins. Oncogene. 16:2265-2282.

48. Green, D.R., and Reed, J.C. 1998. Mitochondria and apoptosis. Science. 281:1309-1312.

49. Halestrap, A.P., Kerr, P.M., Javodov, S., and Woodfield, K.Y. 1998. Elucidating the molecular mechanism of the permeability transition pore and its role in the reperfusion injury in the heart. Biochim. Biophys. Acta. 1366:79-94. 